\title{
Estimation of Complexity of Field Contours of Layer Building with the Use of Cell Method of Determining the Fractal Dimension
}

\author{
Yaroslav Garashchenko* \\ National Technical University "Kharkiv Polytechnic Institute", Institute of Mechanical Engineering and Transport, Department of Integrated Technologies \\ of Mechanical Engineering, 2 Kyrpychova St., Kharkiv 61002, Ukraine
}

\begin{abstract}
The results of estimating the geometric complexity of contours in layered building of a product by additive manufacturing are presented. The contour complexity was evaluated on the basis of a statistical analysis of their fractal dimension obtained the cell method. To determine fractal dimension of the contour, measures commensurate with the geometric limitations of layered building were used. Such limitations exist because of layered building peculiarities and technological capabilities of used equipment. Software has been developed to implement a layer-by-layer analysis of the original triangulation 3D model. Approbation of the software was carried out on the basis of models of industrial products. As a result, the possibility of estimating the geometric complexity of the field contours of layered building is confirmed on basis of statistical analysis of distribution characteristics of fractal dimension of the contours.
\end{abstract}

Keywords: additive manufacturing, triangulated model, geometrical complexity, fractal dimension.

\section{Introduction}

Initially at technological preparation of additive manufacturing manufacturability of product is estimated, the choice of production strategy, forecasting of time, prime cost and quality of a product is carried out [1]. A preliminary assessment of the product complexity based on the analysis of original 3D model is necessary for a rational choice of production strategy and equipment [2]. The peculiarity of the processes of additive manufacturing leads to the need for layer-by-layer analysis by estimating the fields of layered building.

The geometric complexity of the product determines, when creating a set of layers, complexity of field contours. The assessment of the contour complexity must be carried out taking into account the existing geometrical restrictions of used technology and equipment.

Experimental studies of the geometrical restrictions of additive manufacturing, i.e. a sufficiently large number of publications are devoted to determining the lower limit size of various elements of product. In [3-5] for SLM, SLS and FDM technologies geometrical restrictions on elements such as holes, cylinders, walls, and fonts were defined.

For example, in [6], a quantitative assessment of the complexity is performed on relative perimeter of visible contour from a given point belonging to it.

One of the known approaches in geometry is the complexity estimation of a curve from its fractal dimension obtained using the cell method [7]. Therefore, the fractal dimension can be used as a measure of contour complexity. It is also assumed that the correct selection of the scale of measurement of the contour, commensurate 
with geometric restrictions, in determining the fractal dimension will allow a reasonable choice of equipment for materialization of the 3D model.

In this paper was considered the scientific hypothesis that statistical analysis of distribution of fractal dimensions of the field contours of layered building obtained by using the cell method will allow science-based determine their geometric complexity and accordingly choose technology for obtaining the product.

Purpose of the work is to study the possibilities of evaluating field contours complexity of layered building on basis of the cell method for determining their fractal dimension, taking into account the geometrical restrictions of used technology and equipment.

\section{Experimental Section}

Implementation of the task of transition from original triangulation 3D model of product to the set of layers (contours of the fields) was carried out in the technological preparation system for the materialization of complex products by additive technologies developed at "Integrated technologies of mechanical engineering" department of NTU "Kharkiv Polytechnic Institute" (Ukraine). This system allows determining the workability of industrial product and efficiency of problems decision of technological preparation of additive manufacture on basis of analysis of polygonal, voxel and layered model. To solve this problem, a subsystem of statistical layer-by-layer analysis has been developed. The screen form of the subsystem is shown in Fig. 1.

The developed subsystem presents the following main features:

$\checkmark$ creation of a layers set with $2 D$ sections (field contours) of product on basis of a polygonal 3D-model (STL-file) taking into account the specified slicing strategy (with constant or variable steps);

$\checkmark$ visualization of current layer, taking into account the image scale and color for outer and inner contours;

$\checkmark$ determination of fractal dimension from a given scale range (scaling) for measuring the contour length;

$\checkmark$ determination of basic statistical characteristics (12 parameters are output in a single table for all layers);

$\checkmark$ statistical analysis and display of distribution histograms of investigated criterion (inclination relative to the Z-axis of face normals in current layer and adjacent angles between straight segments of contour);

$\checkmark$ visualization of analysis results in the form of relative or cumulative distribution function.

The transition from original triangulation model of product to the set of layers is performed according to a procedure developed taking into account the existing works [8-10], which implements the strategy with a constant building step.

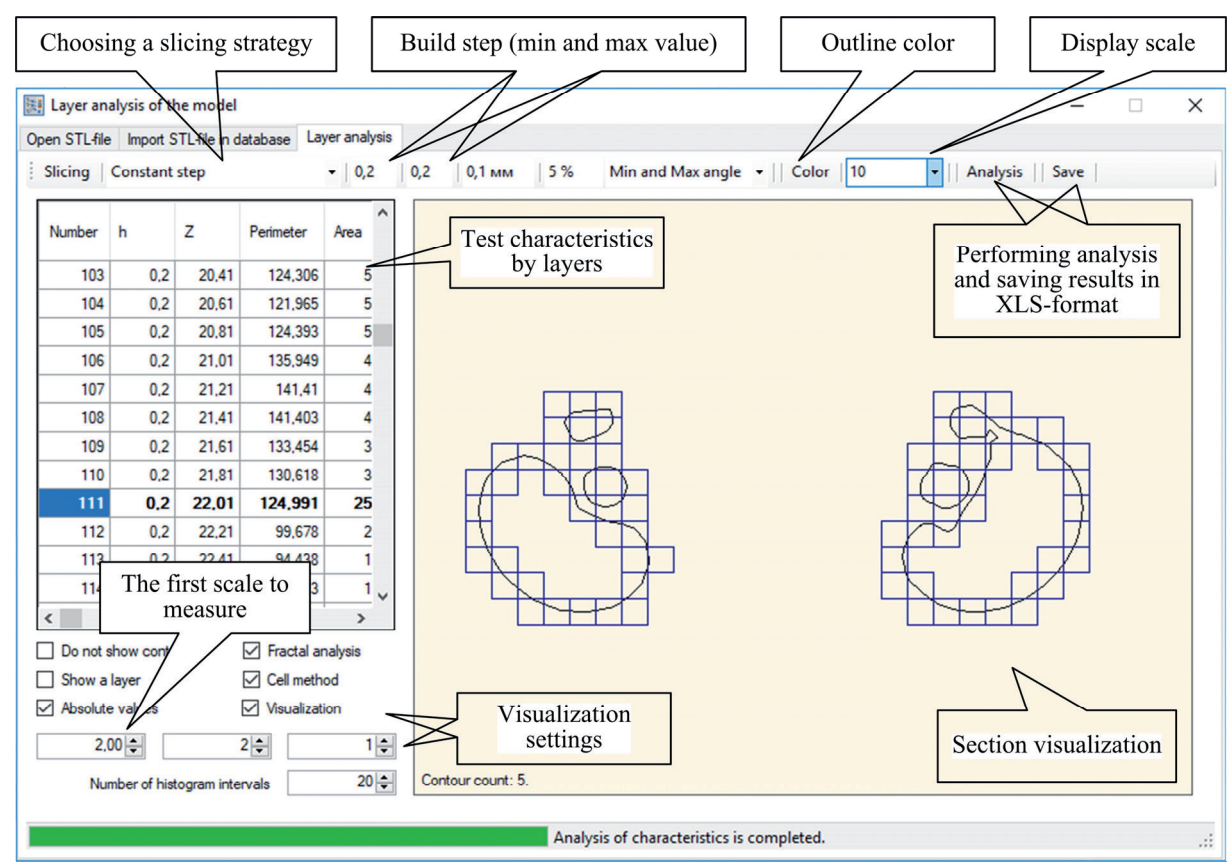

Fig. 1: Screen form of subsystem of layer-by-layer analysis of 3D model.. 


\section{Determining fractal dimension of contour}

A set of layers is formed by cutting the triangulation 3D-model of the product with a set of parallel planes. As a result, closed contours are formed, consisting of straight lines segments. Such segments are obtained as a result of intersection of triangular planar faces of 3D-model by a plane perpendicular to $Z$ axis (which determines the position of layer). In most cases, straight segments relative to total length of contour are sufficiently small. Therefore, the typical task of fractal geometry to determine the curve length using cells with specified dimensions (so-called "measurement scale" $M$ [7]) is fully applicable to field contours of layered building. The result of solving this task is the coefficient of fractal dimension $D$ of contour for a certain scaling range $\Delta M$ (cell size interval).

Taking into account the theoretical premises [11, 12], the fractal dimension $D$ quantitatively estimates the contour complexity, as coefficient of change in detail with change in scale.

In practice, there are necessarily limitations on the minimum and maximum value of measurement scale $M$ [13]. In this paper, the task was to evaluate the complexity of contour taking into account the given geometrical restrictions of technology. Therefore, the value of $M$ used to determine the dimension $D$ was specified in the value range of geometrical restrictions.

The scheme for determining the fractal dimension of contour based on the layer-by-layer analysis of triangulated model is shown in Fig. 2.

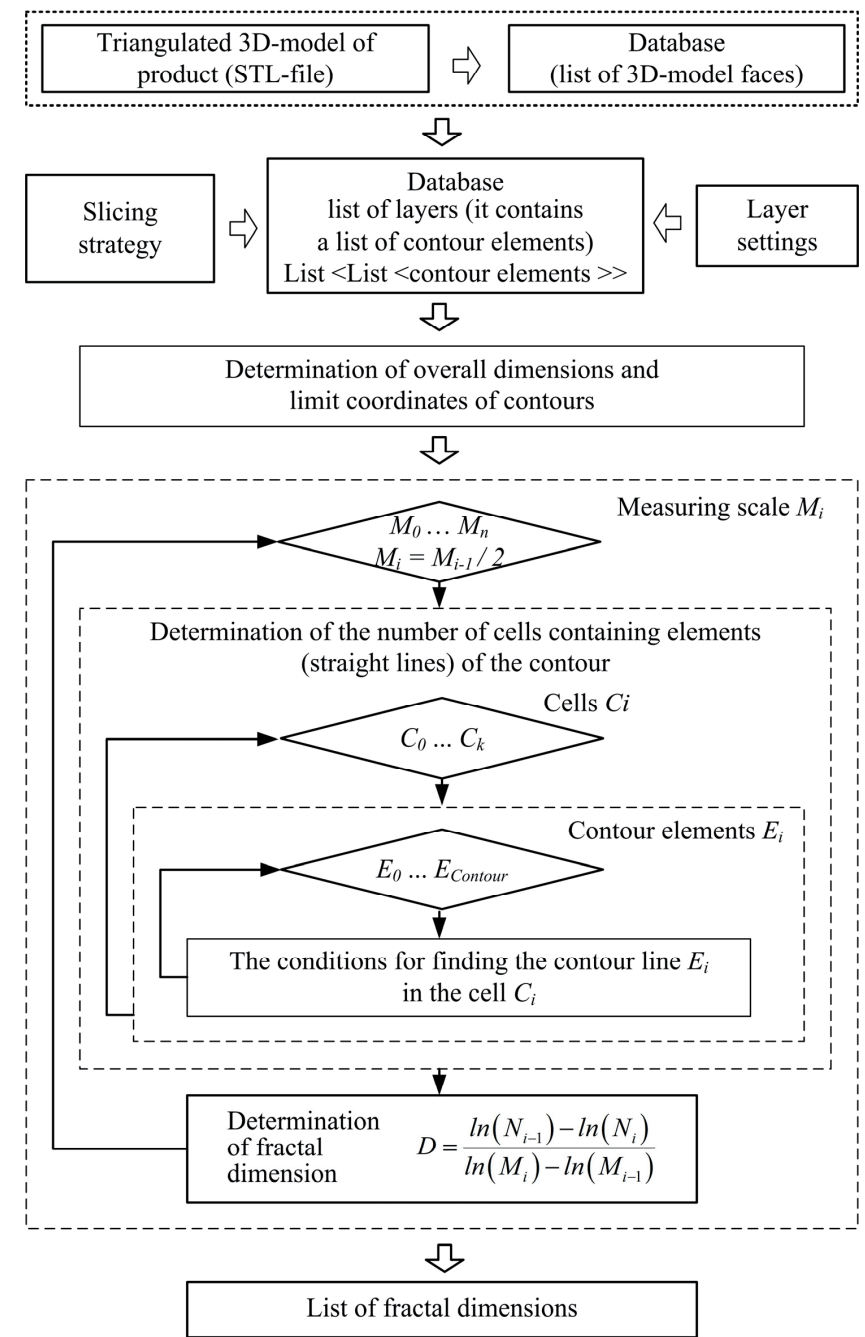

Fig. 2: The scheme for calculating the fractal dimension of contours. 
According to this scheme (Fig. 2), during the measurement of contour length $L$, the minimum number of scales $N$ (cell size $M$ ) covering the entire contour [7] was determined (an example of visualization of measurement procedure is shown in Fig. 3).

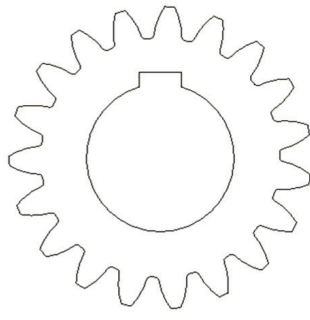

(a)

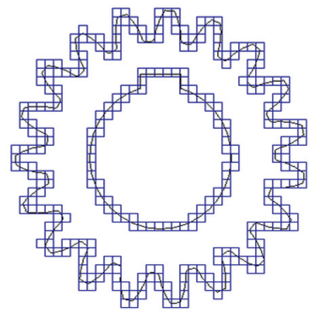

(b)
Fig. 3: Visual analysis of estimation of fractal dimension of contour for model of gear. (a) Original contour. (b) Cell contour.

When the scale $M$ is repeatedly changed, the measurement process is repeated. With respect to cell method, scale is a cell (square) of size $M$. The scale is a priori assumed to be much smaller than length of measured contour $(M<<L)$. The amount of $N$ will increase as the value of $M$ decreases (for a full coverage of contour, a larger number of smaller scales is required).

The fractal dimension is determined from the following relationship [7]:

$$
D=\frac{\ln \left(N_{i-1}\right)-\ln \left(N_{i}\right)}{\ln \left(M_{i}\right)-\ln \left(M_{i-1}\right)},
$$

where $M_{i}$ - measure (circle radius) for i-th measurement of contour length; $N_{i}$ - number of scales (circles with radius $M_{i}$ ) covering the contour.

As a result, the coefficient $D$ obtained from (1) can take not an integer value, and indicate the degree of filling of plane by the contour [14].

With respect to the contour consisting of straight lines, its complexity and, correspondingly, the fractal dimension will depend on scaling $\Delta M=M_{i 1}-M_{i}$. When measuring a real contour using a measure $M \rightarrow 0$, then length $L=$ const. In this case, based on the dependence (1), the fractal dimension will correspond to the Euclidean dimension of contour $D=1$. But given when the triangulation error value of CAD model $\left(\Delta_{t r} \rightarrow 0\right)$ is decrease with respect to complex products (containing curve-based surfaces) contour length is increase
$L \rightarrow$ Lideal. Consequently, fractal dimension of contour obtained by cutting the plane curve-based surfaces will always be $D>1$ (provided that cell size $M$ is not smaller than contour sizes $S c$ ).

The possibility of estimating the fractal dimensions of contours was studied using the example of test models of simple geometric bodies and industrial products (Fig. 4). Slicing of the models was carried out according to the strategy with a constant building step. The layer depth was set $h_{L}=1 \div 3 \mathrm{~mm}$. Such an overestimated value of $h_{L}$ was chosen for reasons of decreasing computational time.

The initial measure $M_{1}$ was set relative to the arithmetical mean of overall dimensions of contour $\left.M_{1}=\left\{S_{C} / 10, S_{C} / 5\right)\right\}$ or in absolute values $M_{1}=\{1$, 2\} $\mathrm{mm}$. The subsequent values of the measures were set according to a decreasing geometric progression $M_{i}=q \cdot M_{i-1}$ with ratio $q=2^{-1}$. The measurements number of the contour length was made up to 3 times in order to obtain a wider scaling range $\Delta M$.

The results of model calculations for determining the fractal dimension of contours for test industrial products (Fig. 4) are given in Table 1.

A comparative analysis of the results was performed by using the arithmetical mean of coefficients $\bar{D}$ obtained within the scaling range.

The coefficients $D$ obtained by model calculations (Table 1) correspond to the theoretical premises.

The use of relative values of $M$ made it possible to obtain a fractal dimension regardless of contour scale (Table 1). In this case, the values of $D$ are obtained relative, which can be compared. This circumstance makes it possible to perform a comparative analysis of contours by their complexity. When setting the absolute values of $M$ (Table 1), it becomes possible to evaluate contour taking into account the geometrical restrictions of used technology.

The data presented in Table. 1 the analysis results of fractal dimension of contours for models of industrial products confirm the supposed relationship between the contour complexity and the coefficient $\bar{D}_{A b s}$. The models of the body, gears and panels (Figures $4 a-c$ ) have the most geometrically simple contours of the layered building field. The souvenir model (Figure $4 \mathrm{~g}$ ) has the most complex contours. Consequently, this model will present increased requirements on the technological capabilities of equipment for its production. Under the technological capabilities of 


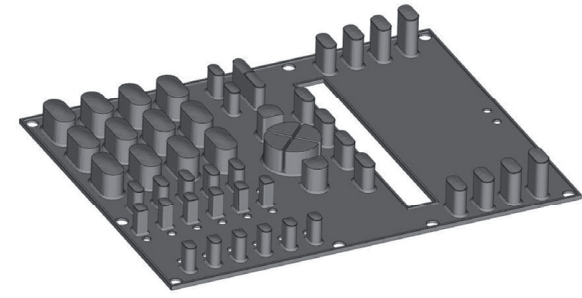

$a$

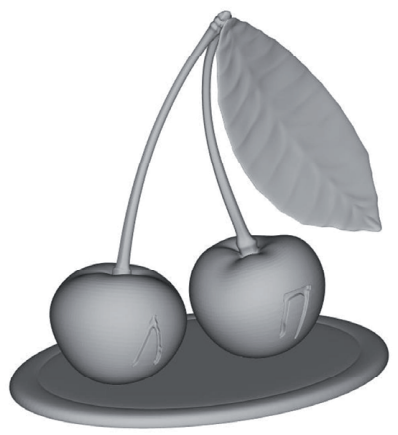

$d$

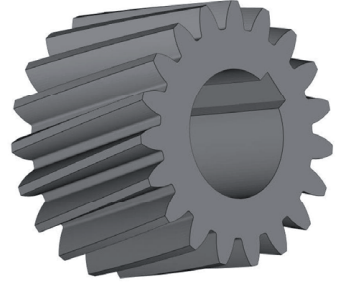

$b$

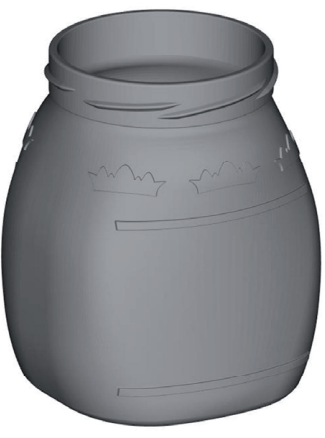

$e$

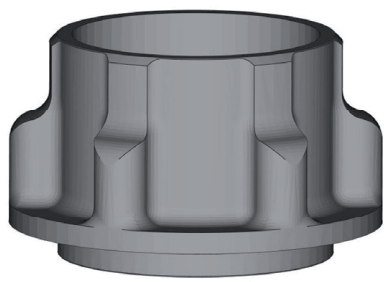

$c$

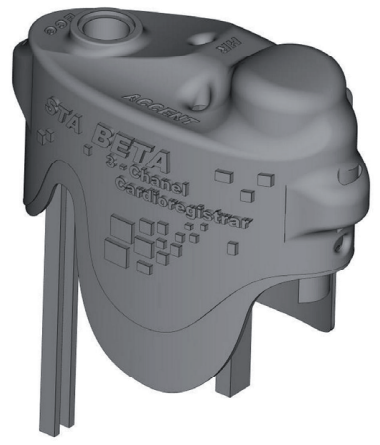

$i$

Fig. 4: Test 3D models. (a) Panel. (b) Gear. (c) Body. (d) Souvenir. (e) Capacity. (i) Cover.

Tab. 1: The fractal dimension of contours when using cells with relative and absolute dimensions.

\begin{tabular}{|c|c|c|c|c|c|c|}
\hline Model (overall dimensions, mm) & $M_{1}$ & $D_{\text {Rel }}$ & $\bar{D}_{R e l}$ & $M_{1, \mathrm{~mm}}$ & $D_{A b s}$ & $\bar{D}_{A b s}$ \\
\hline \multirow{2}{*}{$\begin{array}{l}\text { Panel } \\
(152 \times 196 \times 20)\end{array}$} & $S_{c} / 10$ & $0.868 \div 1.463$ & 1.230 & 2 & $0.901 \div 1.122$ & 1.006 \\
\hline & $S_{c} / 5$ & $0.925 \div 1.417$ & 1.228 & 1 & $0.997 \div 1.004$ & 1.001 \\
\hline \multirow{2}{*}{$\begin{array}{l}\text { Gear } \\
(86 \times 86 \times 60)\end{array}$} & $\mathrm{S}_{\mathrm{C}} / 10$ & $1.100 \div 1.136$ & 1.114 & 2 & $0.993 \div 1.013$ & 1.004 \\
\hline & $S_{c} / 5$ & $1.196 \div 1.232$ & 1.218 & 1 & $0.993 \div 1.010$ & 1.002 \\
\hline \multirow{2}{*}{$\begin{array}{l}\text { Body } \\
(210 \times 210 \times 125)\end{array}$} & $S_{c} / 10$ & $1.165 \div 1.357$ & 1.218 & 2 & $0.995 \div 1.012$ & 1.001 \\
\hline & $S_{c} / 5$ & $1.181 \div 1.441$ & 1.266 & 1 & $0.998 \div 1.005$ & 1.000 \\
\hline \multirow{2}{*}{$\begin{array}{l}\text { Souvenir } \\
(73 \times 51 \times 70)\end{array}$} & $S_{c} / 10$ & $0.918 \div 1.306$ & 1.096 & 2 & $0.942 \div 1.303$ & 1.112 \\
\hline & $S_{c} / 5$ & $0.850 \div 1.264$ & 1.035 & 1 & $0.792 \div 1.164$ & 1.044 \\
\hline \multirow{2}{*}{$\begin{array}{l}\text { Capacity } \\
(102 \times 94 \times 125)\end{array}$} & $S_{c} / 10$ & $1.186 \div 1.321$ & 1.265 & 2 & $1.000 \div 1.171$ & 1.112 \\
\hline & $S_{c} / 5$ & $1.096 \div 1.316$ & 1.180 & 1 & $1.000 \div 1.010$ & 1.001 \\
\hline \multirow{2}{*}{$\begin{array}{l}\text { Cover } \\
(84 \times 101 \times 43)\end{array}$} & $S_{c} / 10$ & $1.000 \div 1.287$ & 1.131 & 2 & $0.975 \div 1.214$ & 1.018 \\
\hline & $\mathrm{S}_{\mathrm{c}} / 5$ & $1.000 \div 1.453$ & 1.180 & 1 & $0.992 \div 1.116$ & 1.014 \\
\hline
\end{tabular}

equipment in this context is meant the allowable minimum dimensions of the product elements.

The analysis of the $D_{A b s}$ values showed the existence of relationship $D_{A b s}=f(M)$. Assuming that the best contour reproduction will be realized for a contour with a fractal dimension $D=1$. Therefore, this relationship is further considered, which is necessary to evaluate the effect of geometrical restrictions on the accuracy of obtaining a contour.

\section{The relationship between measure and fractal dimension of the contour. Discussion of the results}

The influence of scaling range on fractal dimension of the contours was determined by using the cover model as an example (Fig. 4 i). The dependence 
$D_{A b s}=f(M)$ obtained is shown in Fig. 5.

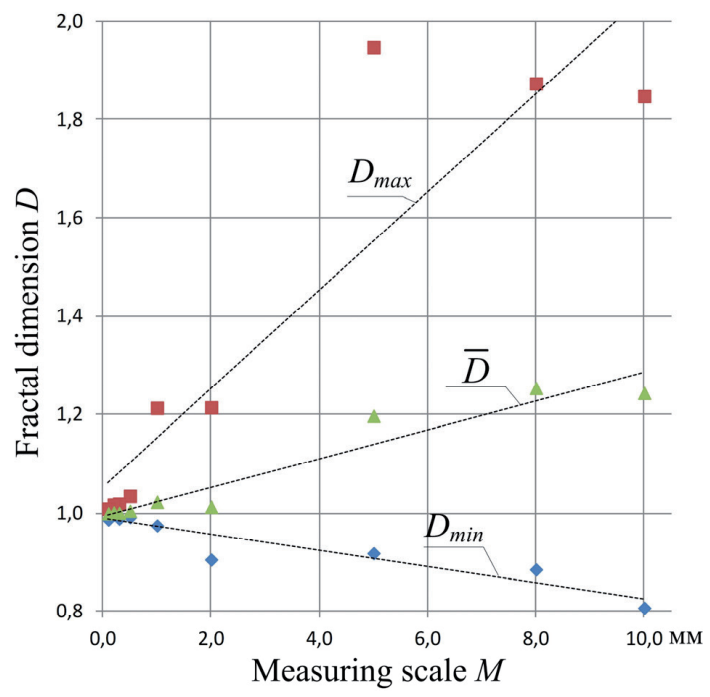

Fig. 5: Dependence of fractal dimension of the contours on scale of measurement for the cover model (Fig. 4 i).

For the cover model, the minimum value of $D_{A b s}$ is significantly less than the Euclidean dimension ( $D_{A b s}<E=1$ ) over the entire interval $M$ (for all cell sizes). This circumstance indicates the presence of small contours with dimensions commensurate with scale $M$. The maximum value of $D_{A b s}$ varies over a wide range of values $-1.009 \div 1.948$. The average value $\bar{D}_{\text {Abs }}$ in smaller range of values is $1.000 \div 1.253$.

The regularities of the obtained interrelation $D_{A b s}=f(M)$ for the cover model (Figure 5) are common for the considered models of products. First, an increase in $M$ leads to an increase in $D_{A b s .}$ Secondly, the dependence can be described by a sectionally function. This feature is regularity, as it is observed for all considered models of industrial products. The explanation for such sharp changes in fractal dimension of $D_{A b s}$ can be the small size of some contours or the effect of multiple overlapping of cells on straight sections of contour. This feature does not make it possible to reveal the functional dependence of both the general and the individual product model. Accordingly, when choosing the technical characteristics of equipment, it is necessary to determine the fractal dimension in the scaling region $\Delta M$ corresponding to the geometrical restriction range. Based on the revealed regularities, it is possible to make the assumption that the minimum value of fractal dimension will indicate the most rational choice of technology and equipment for materialization of the given 3D-model.

\section{Conclusions}

Statistical analysis of distribution of fractal dimension of field contours by cell method allows determining their geometric complexity for layered building.

Setting the cell size $M$ relative to the arithmetic average of the overall dimensions of the contour allow to obtain a fractal dimension regardless of contour scale. With this approach, the resulting dimensions $D_{R e l}$ are applicable for performing a comparative analysis of the contour complexity.

Minimizing the fractal dimension will allow to determine the scaling, which should correspond to the rational values of the geometric restrictions of the chosen technology and equipment for materializing the given 3D model.

\section{References and Notes}

[1] Zhang, Y., Bernard, A. (2014). AM Feature and Knowledge Based Process Planning for Additive Manufacturing in Multiple Parts Production Context. In Proceedings of 25th Annual International Solid Freeform Fabrication Symposium, 1259-1276.

[2] Xie, X. (2003). Design for manufacture and assembly. Utah Dept Mechanical, 12, from http://home.utah. edu/ u0324774/pdf/DFMA.pdf.

[3] Thomas, D. (2009). The development of design rules for selective laser melting. Thesis (PhD). University of Wales, Cardiff, 318, from http://hdl.handle.net/10369/913.

[4] Adam, G.A.O., Zimmer, D. (2014). Design for additive manufacturing - element transitions and aggregated structures. CIRP Journal of Manufacturing Science and Technology, 7 (1), 20-28.

[5] Kranz, J., Herzog, D., Emmelmann, C. (2015). Design guidelines for laser additive manufacturing of lightweight structures in TiAl6V4. Journal of Laser Applications, 27, 14001-14016.

[6] Psarra, S., Grajewski, T. (2001). Describing shape and shape complexity using local properties. Proceedings, 3rd International Space Syntax Symposium 2001, Atlanta, USA, 28.1-28.16. from http://discovery.ucl.ac.uk/id/ eprint/1320681.

[7] Balkhanov, V.K. (2013). Fundamentals of fractal geometry and fractal calculus. Ulan-Ude: Publishing house of the Buryat State University, 224 [in Russian].

[8] Pandey, P.M., Reddy, N.V., Dhande, S.G. (2003). Slicing procedures in layered manufacturing: a review. Rapid 
Prototyping Journal, Vol. 9, Issue: 5, 274-288.

[9] Abdurayimov, L.N. (2009). Adaptive layering of the original $3 \mathrm{D}$ model of the product in rapid prototyping and manufacturing techniques. Scientific notes of the Crimean Engineering and Pedagogical University. Engineering science, Simferopol: SRC KIPU, Iss. 18, 15-20 [in Russian].

[10] Topçu, O., Taşcıoğlu, Y., Ünver, H.Ö. (2011). A Method for Slicing CAD Models in Binary STL Format. 6th International Advanced Technologies Symposium (IATS'11), 141-145.

[11] Mandelbrot, B. (1983).The fractal geometry of nature. Macmillan, 468.

[12] Albers, A., Gerald, A.L. Mandelbrot, B. (2008). In his own words. Mathematical people: profiles and interviews. Wellesley, Mass: AK Peters, 214

[13] Bozhokin, S.V, Parshin, D.A. (2001). Fractals and multifractals. Izhevsk: SIC "Regular and chaotic dynamics", 128 [in Russian].

[14] Falconer, K. (2003). Fractal Geometry: Mathematical Foundations and Applications. New York, 366.

\section{Biographical notes}

Assoc. Prof. Yaroslav Garashchenko, Ph.D., an Associate Professor of the Department of Integrated Technologies of Mechanical Engineering of National Technical University "Kharkiv Polytechnic Institute" (Ukraine). He was born in 1975. He received a B.S. Degree in Mechanical Engineering and Specialist Degree in Manufacturing Engineering from Kharkiv Polytechnic Institute (Ukraine) in 1997 and 1999 respectively; an Ph.D. Degree in Manufacturing Engineering from National Technical University "Kharkiv Polytechnic Institute" (Ukraine) in 2004. He took part in research projects funded by Ministry of Education and Science of Ukraine and EU grant programs TEMPUS. He was trained at Delcam PIC (Great Britain) in 2008, Technical University of Dresden (Germany) in 2008 and Technical University of Liberec (Czech Republic) in 2009. His research activities concern design; include analysis, optimization, verification, reverse engineering; CAD/CAM methods in manufacturing engineering; additive manufacturing (3D printing); modeling of grinding tools; engineering education. His works within this scope were published in textbooks, a monograph, national and international journals and presented at many scientific conferences.

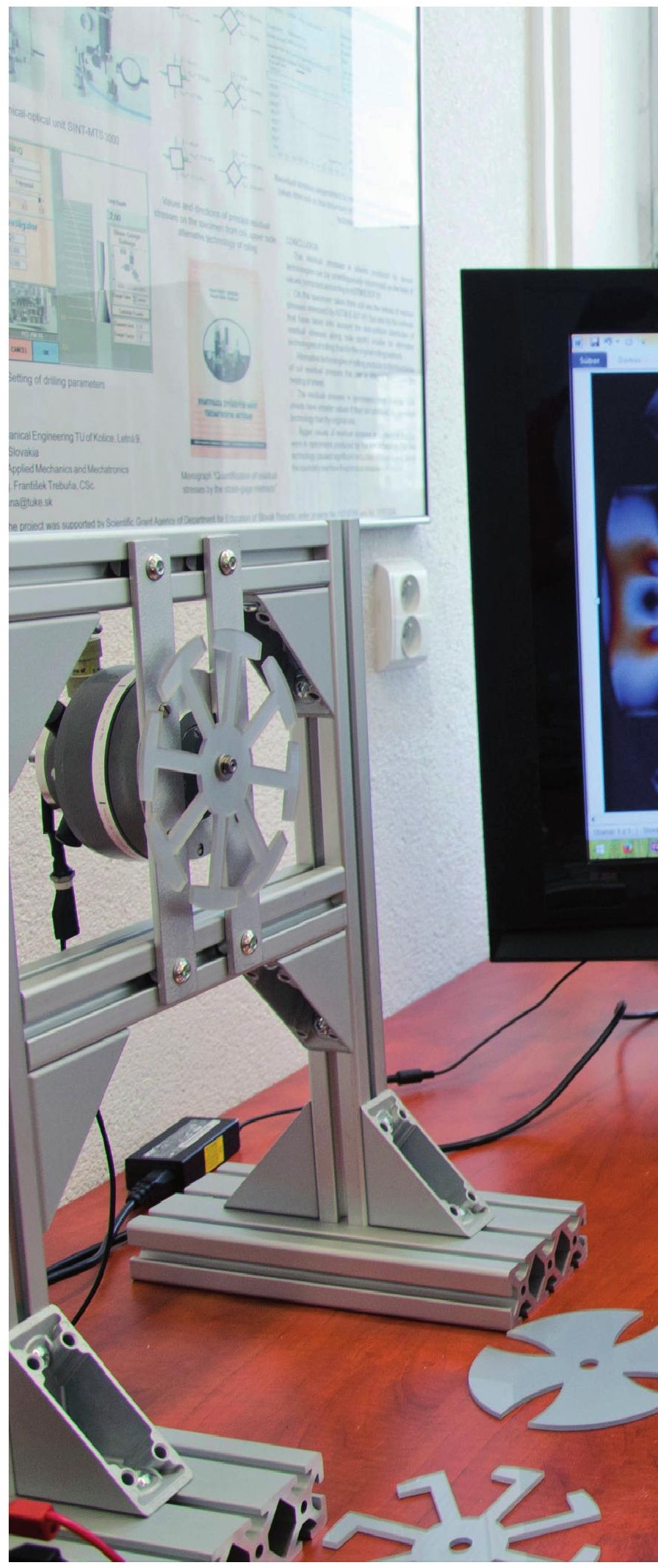


